\title{
Estudo do efeito da diluição nas propriedades microestruturais e magnéticas de ferritas $\mathrm{Mn}_{0,65} \mathrm{Zn}_{0,35} \mathrm{Fe}_{2} \mathrm{O}_{4}$ por reação de combustão
}

\section{(Study of the effect of dilution on the microstructural and magnetic properties of $\mathrm{Mn}_{0.65} \mathrm{Zn}_{0.35} \mathrm{Fe}_{2} \mathrm{O}_{4}$ ferrites by combustion reaction)}

\author{
R. L. P. Santos ${ }^{1}$, V. C. S. Diniz ${ }^{2}$, R. H. G. A. Kiminami ${ }^{3}$, D. R. Cornejo ${ }^{4}$, A. C. F. M. Costa ${ }^{2}$ \\ ${ }^{I}$ Departamento de Engenharia de Materiais, UFRN, Natal, RN, Brasil 59078-970 \\ ${ }^{2}$ Departamento de Engenharia de Materiais, UFCG, Campina Grande, PB, Brasil \\ ${ }^{3}$ Departamento de Engenharia de Materiais, UFSCar, S. Carlos, SP, Brasil \\ ${ }^{4}$ Instituto de Física, USP, S. Paulo, SP, Brasil \\ raffitasantos8@hotmail.com,veronicacristhina@hotmail.com,ruth@ufscar.br,cornejo@if.usp.br, \\ c_r_i_s_2005@yahoo.com.br
}

\begin{abstract}
Resumo
As ferritas Mn-Zn vêm sendo utilizadas em diversas áreas tecnológicas por apresentarem propriedades atrativas no âmbito técnico-científico. Este trabalho teve como objetivo sintetizar ferritas $\mathrm{Mn}_{0,65} \mathrm{Zn}_{0,35} \mathrm{Fe}_{2} \mathrm{O}_{4}$ por reação de combustão e avaliar o efeito da diluição dos reagentes em água destilada sobre as características microestruturais e magnéticas. As amostras foram caracterizadas por difração de raios X, microscopia eletrônica de varredura e transmissão, temperatura de Curie e por medidas magnéticas. Foi observado que o aumento da diluição em água durante a síntese por reação de combustão foi favorável para obtenção da ferrita $\mathrm{Mn}_{0,65} \mathrm{Zn}_{0,35} \mathrm{Fe}_{2} \mathrm{O}_{4}$ monofásica. Também favoreceu a diminuição do tamanho de aglomerado e aumento da temperatura de Curie com valor máximo de magnetização de saturação de 62 emu/g para a amostra diluída na proporção 1:10. Palavras-chave: ferrita $\mathrm{Mn}-\mathrm{Zn}$, reação de combustão, caracterização magnética.
\end{abstract}

Abstract

The Mn-Zn ferrites have been used in various technological areas, due to their attractive properties in technical and scientific senses. This paper aimed to synthesize $\mathrm{Mn}_{0.65} \mathrm{Zn}_{0.35} \mathrm{Fe}_{2} \mathrm{O}_{4}$ ferrites by combustion reaction and evaluate the effect of dilution of the reactants in distilled water on the microstructural and magnetic characteristics. The samples were characterized by $X$-ray diffraction, scanning and transmission electron microscopy, Curie temperature and magnetic measurements. It was observed that increasing dilution with water during synthesis by combustion reaction was favorable for obtaining monophasic $\mathrm{Mn}_{0.65} \mathrm{Zn}_{0.35} \mathrm{Fe}_{2} \mathrm{O}_{4}$ ferrite. It also favored a decrease in the agglomerate size and an increase in Curie temperature with maximum value of saturation magnetization of $62 \mathrm{emu} / \mathrm{g}$ for the sample diluted in 1:10 ratio.

Keywords: $M n-Z n$ ferrite, combustion reaction, magnetic characterization.

\section{INTRODUÇÃO}

As nanopartículas de ferrita $\mathrm{Mn}-\mathrm{Zn}$ são materiais ferrimagnéticos que em comparação com os seus homólogos em massa apresentam propriedades físico-químicas únicas, como alta estabilidade química, propriedades magnéticas melhoradas, alta resistividade elétrica, baixas perdas por correntes parasitas, biocompatibilidade, entre outras. Em consequência, elas se encontram entre os materiais magnéticos adequados para vários tipos de aplicações nas áreas da eletrônica, interferência eletromagnética, armazenamento de dados e, também, em aplicações biomédicas, como hipertermia e como agentes de contraste em imagens de ressonância magnética [1-3]. Além disso, são também utilizadas na modalidade neuroprotecção ocular em aplicações clínicas de glaucoma [4] e em experimentos in vivo e in vitro, o que confirmou seu excelente desempenho magnético, capacidade termostática e boa biocompatibilidade [5].

$\mathrm{O}$ processamento das ferritas é o mesmo utilizado na tecnologia de processamento cerâmico em geral; industrialmente o mais utilizado é o método de mistura de óxidos convencional. Em escala de laboratório, várias técnicas têm demonstrado eficiência na produção de nanoferritas, a exemplo podem ser citados o método sol-gel [6], coprecipitação [7], precipitação hidrotérmica [8] e reação de combustão [9] como métodos químicos bem consolidados. A síntese por reação de combustão destaca-se, principalmente, por ser uma técnica fácil, segura e rápida para produção de pós cerâmicos, requerer menos energia que os métodos de síntese convencionais e tempo de processamento reduzido para poucos minutos. Associado a tudo isto, a literatura reporta a produção de nanoferritas em escala variada desde 2 até 200 $\mathrm{g} /$ batelada de reação para diferentes aplicações [10-12]. Com base neste contexto, este trabalho teve como objetivo sintetizar ferritas $\mathrm{Mn}_{0,65} \mathrm{Zn}_{0,35} \mathrm{Fe}_{2} \mathrm{O}_{4}$ por reação de combustão e avaliar 
o efeito da diluição dos reagentes em água destilada sobre as características microestruturais e magnéticas.

\section{MATERIAIS E MÉTODOS}

A ferrita $\mathrm{Mn}_{0,65} \mathrm{Zn}_{0,35} \mathrm{Fe}_{2} \mathrm{O}_{4}$ foi obtida por reação de combustão, utilizando como reagentes oxidantes o nitrato de zinco $\left[\mathrm{Zn}\left(\mathrm{NO}_{3}\right)_{2} \cdot 6 \mathrm{H}_{2} \mathrm{O}\right]$, o nitrato de manganês $\left[\mathrm{Mn}\left(\mathrm{NO}_{3}\right)_{2} \cdot 4 \mathrm{H}_{2} \mathrm{O}\right]$ e o nitrato de ferro $\left[\mathrm{Fe}\left(\mathrm{NO}_{3}\right)_{3} \cdot 9 \mathrm{H}_{2} \mathrm{O}\right] \mathrm{e}$ como agente redutor utilizou-se a glicina $\left(\mathrm{C}_{2} \mathrm{H}_{5} \mathrm{NO}_{2}\right)$. A composição inicial da solução foi baseada na valência total dos reagentes oxidantes e redutores utilizando conceitos da química dos propelentes e explosivos [13], de forma que se estabeleça a estequiometria da fase de interesse. A quantidade de reagente total (RT) para cada diluição em água destilada $\left(\mathrm{H}_{2} \mathrm{O}\right)$ apresentou os valores de 1:0, 1:3,5, 1:7 e 1:10 (RT:H $\left.\mathrm{O}\right)$; o recipiente utilizado para síntese foi um cadinho de sílica vítrea com capacidade de $200 \mathrm{~mL}$. As misturas dos reagentes com e sem diluição foram submetidas ao aquecimento externo em resistência espiral a uma temperatura (na resistência) de aproximadamente $600{ }^{\circ} \mathrm{C}$, até atingir a autoignição e consequentemente a combustão. Após a obtenção, as amostras
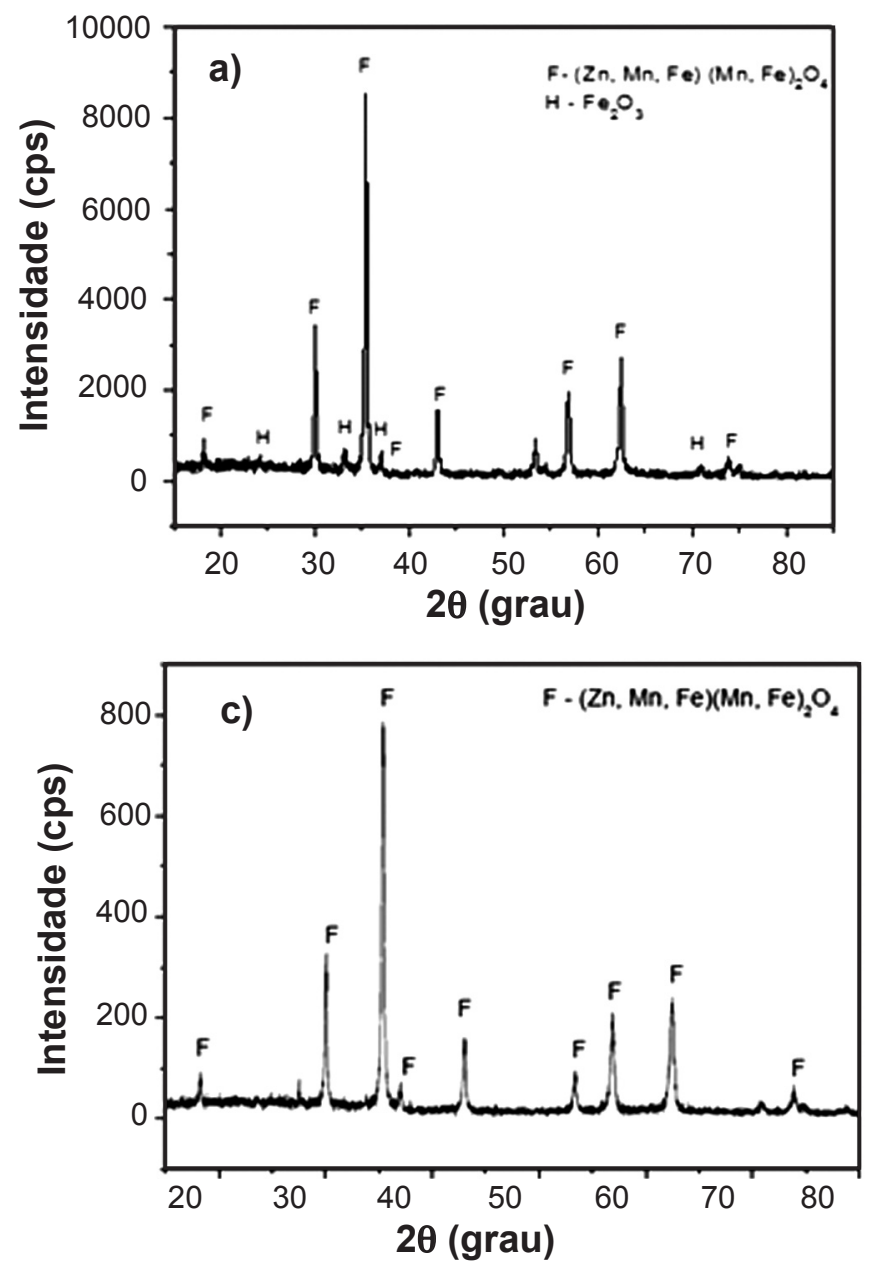

foram desaglomeradas em peneira malha 325 mesh (abertura de $44 \mu \mathrm{m})$ e submetidas à caracterização.

As amostras foram caracterizadas por difração de raios X em um difratômetro Shimadzu (XRD 6000) utilizando fonte de radiação monocromática $(\mathrm{CuK} \alpha, \lambda=1,541 \AA$ Á). $\mathrm{O}$ aspecto morfológico foi analisado por meio de um microscópio eletrônico de varredura (MEV), Philips, XL30 FEG. Para a análise morfológica por microscopia eletrônica de transmissão (MET) foi utilizado um microscópio Philips, EM420 (120 $\mathrm{kV}$ ). Os ciclos de histerese magnética ( $\mathrm{M} \mathrm{x} \mathrm{H} \mathrm{-} \mathrm{magnetização}$ $\mathrm{x}$ campo magnético) das amostras estudadas foram obtidos por um magnetômetro de gradiente alternado (AGM). Por meio das curvas $\mathrm{Mx} \mathrm{H}$, foi possível determinar os valores de alguns parâmetros magnéticos, tais como: campo coercitivo (Hc), magnetização remanescente $(\mathrm{Mr})$ e magnetização de saturação (Ms). A temperatura de Curie foi determinada por análise termomagnética (ATM) em uma taxa de aquecimento de $0,5^{\circ} \mathrm{C} / \mathrm{s}$ em atmosfera de argônio.

\section{RESULTADOS E DISCUSSÃO}

A Fig. 1 ilustra os difratogramas de raios $\mathrm{X}$ das amostras
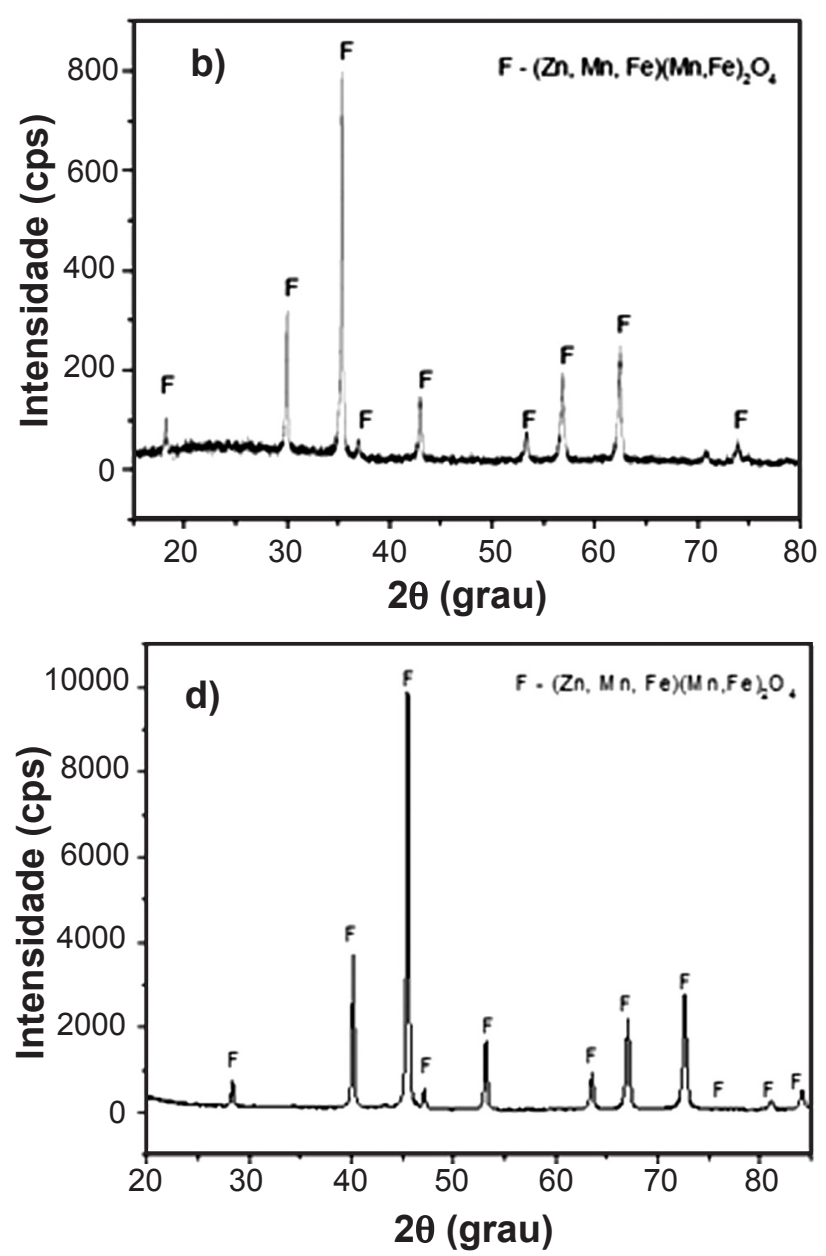

Figura 1: Difratogramas de raios X das amostras de ferrita Mn-Zn preparadas por reação de combustão utilizando glicina como combustível nas proporções de RT: $\mathrm{H}_{2} \mathrm{O}$ de (a) 1:0, (b) 1:3,5, (c) 1:7, (d) 1:10.

[Figure 1: X-ray diffraction patterns of samples of Mn-Zn ferrite prepared by combustion using glycine as a fuel in the proportions of total reagent: $\mathrm{H}_{2} \mathrm{O}$ of (a) $1: 0$, (b) $1: 3.5$, (c) $1: 7$, (d) $\left.1: 10.\right]$ 

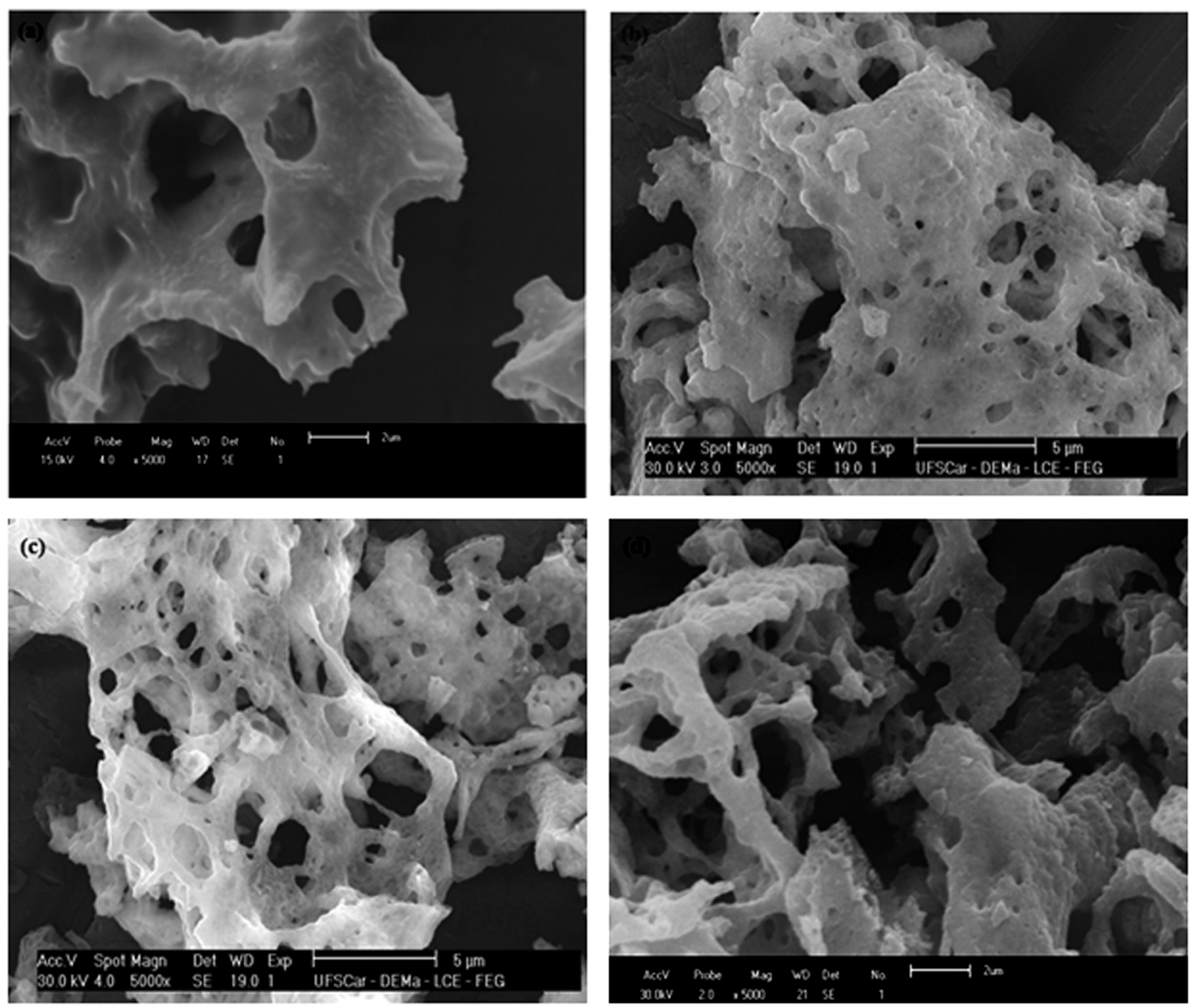

Figura 2: Características morfológicas obtidas por microscopia eletrônica de varredura para ferrita $\mathrm{Mn}_{0,65} \mathrm{Zn}_{0,35} \mathrm{Fe}_{2} \mathrm{O}_{4}$ preparada utilizando glicina como combustível, com diluição em água destilada de (a) 1:0, (b) 1:3,5, (c) 1:7 e (d) 1:10.

[Figure 2: Morphological characteristics obtained by SEM for $\mathrm{Mn}_{0.65} \mathrm{Zn}_{0.35} \mathrm{Fe}_{2} \mathrm{O}_{4}$ ferrite prepared using glycine as a fuel with dilution in distilled water of (a) 1:0, (b) 1:3.5, (c) 1:7, and (d) 1:10.]

de $\mathrm{Mn}_{0,65} \mathrm{Zn}_{0,35} \mathrm{Fe}_{2} \mathrm{O}_{4}$ obtidas com glicina. Para as amostras sintetizadas sem diluição na proporção 1:0 (Fig. 1a), observou-se que além da presença da fase majoritária cúbica do espinélio $\mathrm{Mn}_{0,65} \mathrm{Zn}_{0,35} \mathrm{Fe}_{2} \mathrm{O}_{4}$ (arquivo padrão JCPDF 897554), ocorreu a formação de traços de hematita $\left(\mathrm{Fe}_{2} \mathrm{O}_{3}\right)$ como fase secundária (JCPDF 89-8103). Para as amostras com diluição, observou-se que houve apenas a formação da fase majoritária cúbica do espinélio (JCPDF 89-7554). Isso indicou que a diluição foi favorável para a formação de fase única do espinélio. Este comportamento do favorecimento de amostras monofásicas com o aumento da diluição pode ser explicado pelo fato de que, quando se tem um sistema com alta diluição na presença da glicina, aumenta-se a sua solubilidade, e isto distancia os íons presentes, aumentando o caminho para difusão [14]. Além disso, o sistema não necessita capturar oxigênio atmosférico e dissipa menos energia, favorecendo o aumento da temperatura de combustão, impedindo a mudança de estado de ionização do $\mathrm{Mn}^{2+}$ para $\mathrm{Mn}^{3+}[14]$.

A Fig. 2 ilustra a morfologia das amostras de $\mathrm{Mn}_{0,65} \mathrm{Zn}_{0,35} \mathrm{Fe}_{2} \mathrm{O}_{4}$ preparadas nas proporções 1:0, 1:3,5; 1:7 e 1:10 de RT:H $\mathrm{H}_{2} \mathrm{O}$. A partir das micrografias foi possível observar de forma geral que as amostras apresentaram aspecto poroso, constituídas por aglomerados não densos e de formato irregular. Foi percebida uma alta porosidade, o que possivelmente pode ser atribuída à maior liberação dos gases durante a combustão proporcionada pelo comportamento da glicina como combustível na reação, uma vez que a natureza da aglomeração é regida por entalpia e temperatura da chama gerada durante a combustão, que por sua vez é dependente da natureza do combustível e da relação agente oxidante/agente redutor.

A Fig. 3 ilustra as micrografias obtidas por MET das amostras de $\mathrm{Mn}_{0,65} \mathrm{Zn}_{0,35} \mathrm{Fe}_{2} \mathrm{O}_{4}$ nas proporções 1:0, 1:3,5, 

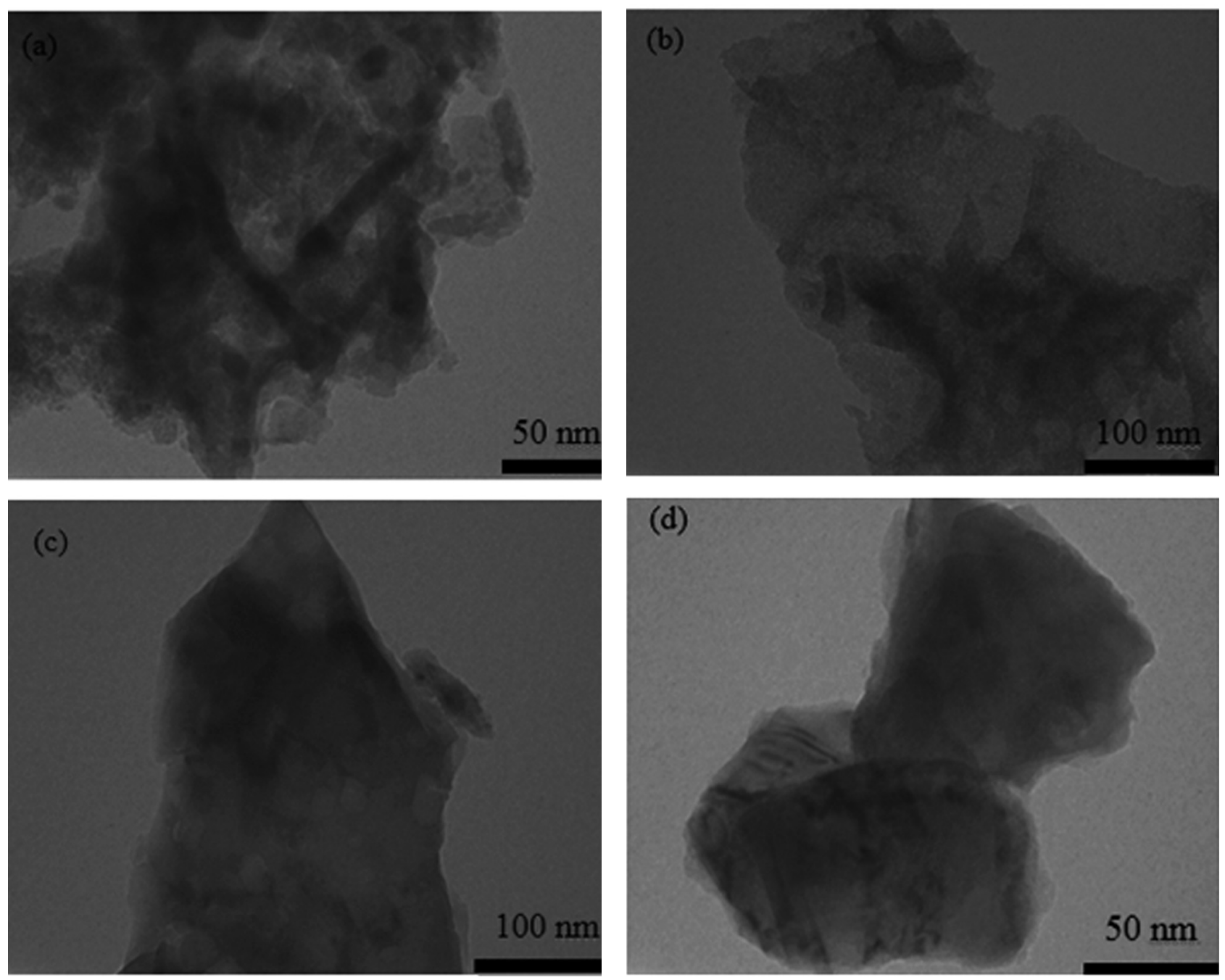

Figura 3: Características morfológicas obtidas por microscopia eletrônica de transmissão para ferrita $\mathrm{Mn}_{0,65} \mathrm{Zn}_{0,35} \mathrm{Fe}_{2} \mathrm{O}_{4}$ preparada com glicina como combustível e diluição em água destilada de (a) 1:0, (b) 1:3,5, (c) 1:7 e (d) 1:10.

[Figure 3: Morphological characteristics obtained by TEM for $\mathrm{Mn}_{0.65} \mathrm{Zn}_{0.35} \mathrm{Fe}_{2} \mathrm{O}_{4}$ ferrite prepared with glycine as a fuel and dilution in distilled water of (a) 1:0, (b) 1:3.5, (c) 1:7, and (d) 1:10.]

1:7 e 1:10 de RT: $\mathrm{H}_{2} \mathrm{O}$. Pode-se observar, de maneira geral, a formação de aglomerados constituídos de pequenas partículas esféricas e tamanhos variados. O tamanho de partícula foi calculado a partir da contagem de 10 partículas obtidas por meio das micrografias; observou-se que para a amostra sem diluição (1:0) o tamanho médio de partícula foi de 30,0 nm e com diluição os tamanhos foram de 19,6, 27,6 e $30,6 \mathrm{~nm}$ para as amostras 1:3,5, 1:7 e 1:10, respectivamente.

A Fig. 4 ilustra a tendência da variação da temperatura de Curie em função da proporção de diluição de ferrita $\mathrm{Mn}-\mathrm{Zn}$ nas proporções 1:0, 1:3,5, 1:7 e 1:10 (RT: $\left.\mathrm{H}_{2} \mathrm{O}\right)$. Observouse que a temperatura de Curie teve uma redução de aproximadamente $30{ }^{\circ} \mathrm{C}$, quando se compara a amostra sem diluição (1:0) com a amostra com diluição 1:3,5. A partir da amostra com proporção de 1:3,5, observou-se um aumento significativo da temperatura de Curie com o aumento da diluição. O comportamento do aumento proporcional da temperatura de Curie, quando da adição da diluição, pode ser justificado pelo sistema ter sido monofásico (sem

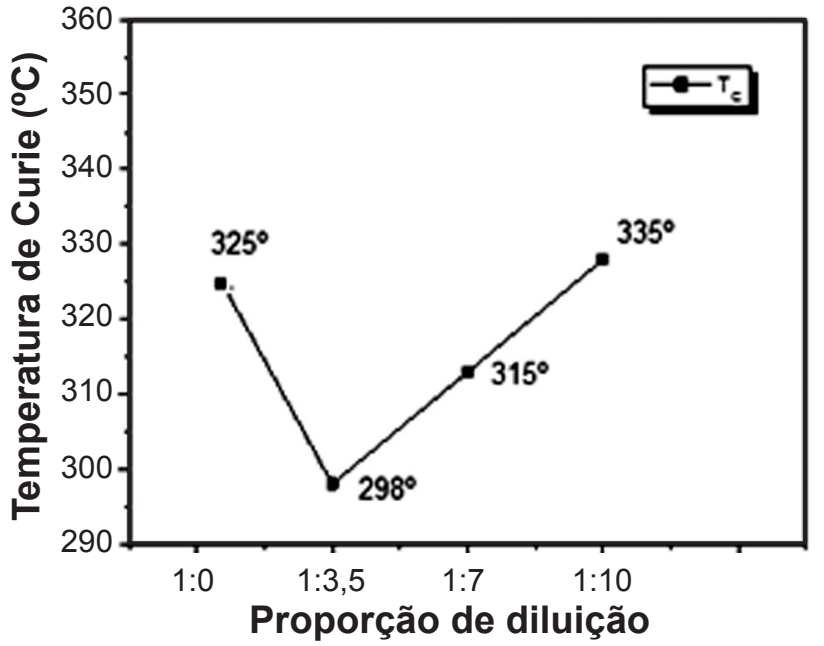

Figura 4: Variação da temperatura de Curie em função da diluição em água destilada.

[Figure 4: Curie temperature variation as a function of dilution in distilled water.] 

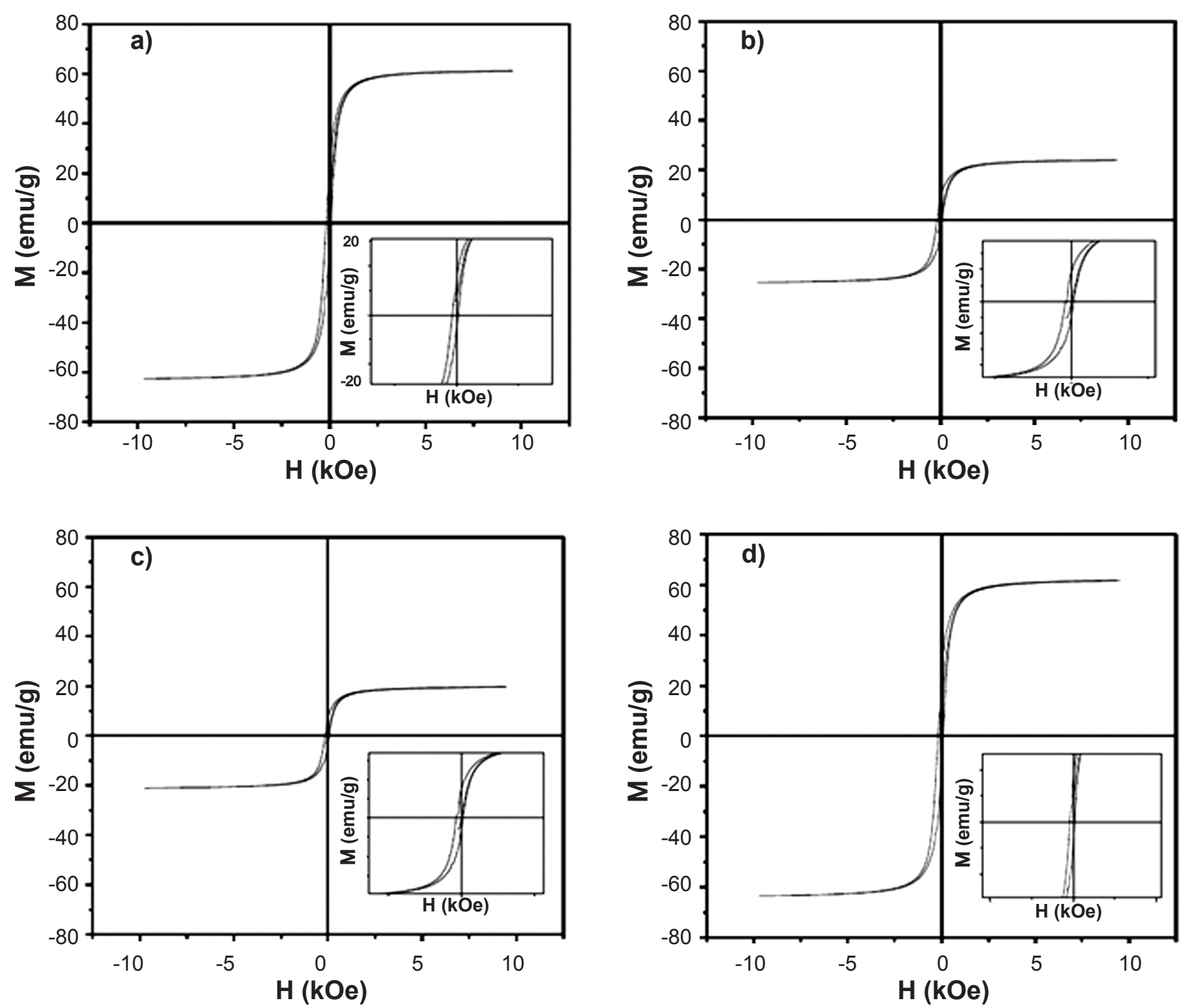

Figura 5: Histerese magnética para as ferritas $\mathrm{Mn}_{0,65} \mathrm{Zn}_{0,35} \mathrm{Fe}_{2} \mathrm{O}_{4}$ preparadas com diluição em água destilada de (a) 1:0, (b) $1: 3,5$, (c) $1: 7$ e (d) $1: 10$.

[Figure 5: Magnetic hysteresis for $\mathrm{Mn}_{0.65} \mathrm{Zn}_{0.35} \mathrm{Fe}_{2} \mathrm{O}_{4}$ ferrites prepared with dilution in distilled water of (a) 1:0, (b) 1:3.5, (c) $1: 7$, and (d) $1: 10$.

presença de hematita). Gama [15], quando estudou o sistema $\mathrm{Mn}_{\mathrm{x}} \mathrm{Zn}_{1-\mathrm{x}} \mathrm{Fe}_{2+\mathrm{x}} \mathrm{O}_{4}$, observou que a temperatura de Curie aumentou com a elevação do teor de óxido de ferro de 442 para $511^{\circ} \mathrm{C}$, e diminuiu quando houve o aumento do teor de óxido de zinco de 459 para $410{ }^{\circ} \mathrm{C}$. Segundo o autor, o aumento na temperatura de Curie das amostras foi atribuído à diferença de raio iônico entre o íon $\mathrm{Fe}^{2+} \mathrm{e}$ $\mathrm{Mn}^{2+}$, pois a substituição dos íons de $\mathrm{Mn}^{2+}$ por $\mathrm{Fe}^{2+}$ diminui a distância interatômica na interação $\mathrm{AB}$, tornando-a mais forte, portanto, uma maior temperatura de Curie [15]. Quando o teor de óxido de zinco foi aumentado, visto que o íon $\mathrm{Zn}^{2+}$ não é magnético e apresenta preferência pela ocupação do sítio A (tetraédrico) na temperatura ambiente, ocorreu um deslocamento dos íons magnéticos para o sítio $\mathrm{B}$ (octaédrico) e a interação $\mathrm{AB}$ diminuiu, fazendo com que a temperatura de Curie também diminuísse [15].
A Fig. 5 ilustra a dependência da magnetização $M$ em função do campo magnético aplicado $H$ das amostras de $\mathrm{Mn}_{0,65} \mathrm{Zn}_{0,35} \mathrm{Fe}_{2} \mathrm{O}_{4}$ nas proporções 1:0, 1:3,5, 1:7, 1:10 de $\mathrm{RT}: \mathrm{H}_{2} \mathrm{O}$. Por meio dessas curvas foi possível determinar os valores de alguns parâmetros magnéticos, tais como: campo coercitivo $\left(\mathrm{H}_{\mathrm{c}}\right)$, magnetização remanente $\left(\mathrm{M}_{\mathrm{r}}\right)$ e magnetização de saturação $\left(\mathrm{M}_{\mathrm{s}}\right)$. Observou-se que todos os ciclos de histerese das amostras apresentaram laço estreito característico de materiais magnéticos moles (soft). As amostras com menores laços de histerese e com áreas mais largas foram as preparadas nas proporções 1:3,5 e 1:7 de $\mathrm{RT}: \mathrm{H}_{2} \mathrm{O}$.

A Tabela I apresenta os parâmetros de histerese das amostras de $\mathrm{Mn}_{0,65} \mathrm{Zn}_{0,35} \mathrm{Fe}_{2} \mathrm{O}_{4}$ nas proporções de 1:0, 1:3,5, 1:7 e 1:10 (RT: $\left.\mathrm{H}_{2} \mathrm{O}\right)$. Com base nos dados apresentados na Tabela I, verificou-se que a diluição de 
Tabela I - Parâmetros de histerese para as amostras sintetizadas utilizando glicina como combustível.

[Table I - Hysteresis parameters for the samples synthesized using glycine as a fuel.]

\begin{tabular}{cccccc}
\hline Amostra & $\begin{array}{c}\mathrm{M}_{\mathrm{s}} \\
(\mathrm{emu} / \mathrm{g})\end{array}$ & $\begin{array}{c}\mathrm{M}_{\mathrm{r}} \\
(\mathrm{emu} / \mathrm{g})\end{array}$ & $\begin{array}{c}\mathrm{H}_{\mathrm{c}} \\
(\mathrm{kOe})\end{array}$ & $\mathrm{M}_{\mathrm{r}} / \mathrm{M}_{\mathrm{s}}$ & $\begin{array}{c}\text { Área }(\mathrm{kOe} . \\
\left.\mathrm{emu} \cdot \mathrm{g}^{-1}\right)\end{array}$ \\
\hline $1: 0$ & 61 & 9,0 & 0,176 & 0,1 & 521 \\
$1: 3,5$ & 20 & 9,3 & 0,163 & 0,4 & 161 \\
$1: 7$ & 24 & 9,3 & 0,168 & 0,5 & 193 \\
$1: 10$ & 62 & 9,0 & 0,211 & 0,1 & 512 \\
\hline
\end{tabular}

uma forma geral favoreceu um aumento na magnetização de saturação. Contudo, foi observado um decréscimo no valor da magnetização de saturação de $67 \%$ quando se compara a amostra sem diluição (1:0) com a amostra com diluição 1:3,5. A partir da amostra 1:3,5 observou-se aumento exponencial da magnetização com o aumento da diluição, sendo compatível com os resultados reportados na temperatura de Curie. O mesmo comportamento foi observado em relação ao campo coercitivo e a área do ciclo de histerese. Esse comportamento pode ser justificado pelas características extrínsecas das amostras, como tamanho de partícula, uma vez que influenciam a área de domínio magnético e podem contribuir para o aumento da magnetização, pois quanto maior o tamanho da partícula, mais fácil é o deslocamento da parede de domínios, consequentemente, maior é a magnetização $[16,17]$.

\section{CONCLUSÕES}

O aumento da diluição dos reagentes em água durante a síntese por reação de combustão foi favorável para obtenção da ferrita $\mathrm{Mn}_{0,65} \mathrm{Zn}_{0,35} \mathrm{Fe}_{2} \mathrm{O}_{4}$ monofásica. A temperatura de Curie aumentou com a diluição, alcançando valor máximo de $335^{\circ} \mathrm{C}$, favorecendo uma melhora na resposta magnética, com valor máximo de magnetização de saturação de 62 emu/g para a amostra diluída na proporção 1:10.

\section{AGRADECIMENTOS}

Os autores agradecem ao CNPq e CAPES pelo suporte financeiro.

\section{REFERÊNCIAS}

[1] R. Valenzuela, Phys. Res. Int. 2012 (2012) 1-9.

[2] M. Abbas, Sri Ramulu, B. Parvatheeswara Rao, M.O. Abdel-Hamed, C.G. Kim, J. Alloys Compd. 644 (2015) 774-782.

[3] H. Yang, C. Zhang, X. Shi, H. Hu, X. Du, Y. Fang, Y. Ma, H. Wu, S. Yang, Biomater. 31 (2010) 3667-3673.

[4] M. Jeun, J.W. Jeoung, S. Moon, Y.J. Kim, S. Lee, S.H. Paek, K.-W. Chung, K. H. Park, S. Bae, Biomater. 32 (2011) 387-394.

[5] M. Lin, D. Zhang, J. Huang, J. Zhang, W. Xiao, H. Yu, L. Zhang, J. Ye, Nanotechnology 24 (2013) 255.

[6] S.A. Ebrahimi, S.M. Masoudpanah, J. Magn. Magn. Mater. 357 (2014) 77-81.

[7] Z. Karimi, Y. Mohammadifar, H. Shokrollahi, G.H. Yousef, L. Karimi, J. Magn. Magn. Mater. 361 (2014) 150156.

[8] A. Xia, C. Zuo, L. Chen, C. Jin, J. Magn. Magn. Mater. 332 (2013) 186-191.

[9] M.H. Mahoud, A.M. Elshahawy, S.A. Makhlouf, H.H. Hamdeh, J. Magn. Magn. Mater. 343 (2013) 21-26.

[10] J. Dantas, J.R.D. Santos, F.N. Silva, A.S. Silva, A.C.F.M Costa, Mater. Sci. Forum 775-776 (2013) 115 109.

[11] A.S. Silva, J. Dantas, J.R.D. Santos, R.B.L. Cunha, R.H.G.A. Kiminami, A.C.F.M. Costa, in Anais $20^{\circ}$ Congr. Bras. Eng. Ciên. Mater. (2012) 1558-1565.

[12] D.A. Vieira, "Processamento e caracterização de nanoferritas $\mathrm{Ni}-\mathrm{Zn}$ para desenvolvimento de dispositivos magnéticos moles", Tese Dr., Universidade Federal de Campina Grande, PB (2013).

[13] S.R. Jain, K.C. Adiga, V.P. Vernek, Combust. Flame 40 (1981) 71-79.

[14] R.L.P. Santos, E. Leal, A.T.C. Silva, A.C.F. de Melo Costa, Mater. Sci. Forum 727-728 (2012) 1266-1271.

[15] A.M. Gama, "Efeito das proporções de $\mathrm{Mn} / \mathrm{Zn}$ e $\mathrm{Fe} / \mathrm{Mn}+\mathrm{Zn}$ na temperatura de Curie de ferritas do tipo $(\mathrm{Mn}+\mathrm{Zn})_{1-\mathrm{x}} \mathrm{Fe}_{2+\mathrm{x}} \mathrm{O}_{4}$ ", Diss. Mestrado, Escola Politécnica da Universidade de S. Paulo, S. Paulo (2003).

[16] A.R. Bueno, L.C.F. Gomes, M.C.S. Nóbrega, in Anais $41^{\circ}$ Cong. Bras. Cerâm., S. Paulo, SP (1997) 135-138.

[17] A. Znidarsic, M. Drofenik, IEEE Trans. Magn. 32, 3 (1996) 1941-1945.

(Rec. 15/03/2016, Rev. 07/04/2016, 10/05/2016, Ac. $10 / 05 / 2016)$ 